DOI: $10,4046 / \operatorname{trd} 2011,70,5,423$

ISSN: 1738-3536(Print)/2005-6184(Online)

Tuberc Respir Dis 2011;70:423-427

Copyright(C)2011. The Korean Academy of Tuberculosis and Respiratory Diseases. All rights reserved.

\title{
Endobronchial Metastasis of Epithelioid Sarcoma
}

Seo Yun Kim, M.D., Ji Yeon Lee, M.D., Yeon Joo Lee, M.D., Sung Soo Park, M.D., Hyeon-Kyoung Koo, M.D., Sang-Min Lee, M.D., Ph.D., Jae-Joon Yim, M.D., Ph.D., Seok-Chul Yang, M.D., Ph.D., Chul-Gyu Yoo, M.D., Ph.D., Sung Koo Han, M.D., Ph.D., Young-Soo Shim, M.D., Ph.D., Young Whan Kim, M.D., Ph.D. Division of Pulmonary and Critical Care Medicine, Department of Internal Medicine and Lung Institute, Seoul National University College of Medicine, Seoul, Korea

\begin{abstract}
Epithelioid sarcomas are rare soft tissue sarcomas with a high tumor grade and high local recurrence and metastasis rates. Although the lung is the most common site of metastasis, endobronchial metastasis hasn't been reported yet. We now report a case of epithelioid sarcoma with endobronchial metastasis. A 28-year-old man had recurrent pneumothorax and underwent wedge resection. He presented at our hospital with hemoptysis, dyspnea, and chest pain. Chest computed tomography revealed left pneumothorax, multiple lung nodules and endobronchial lesions at the right lower basal lobe. Bronchoscopy showed a hemorrhagic mass obstructing the bronchus of the right lower basal lobe. Magnetic resonance imaging revealed multiple nodular lesions in the left thigh muscles. The bronchoscopic biopsy of the endobronchial lesion and the muscle biopsy of the thigh showed the same feature epithelioid sarcoma. This is the first case report of an epithelioid sarcoma with endobronchial metastasis that was diagnosed by bronchoscopic biopsy.
\end{abstract}

Key Words: Sarcoma, Epithelioid; Neoplasm Metastasis; Bronchi; Sarcoma, Soft Tissue

\section{Introduction}

Epithelioid sarcomas are very rare soft tissue sarcoma that comprises less than $1 \%$ of all soft tissue sarcomas ${ }^{1}$. In 1970, Enzinger gave the name epithelioid sarcoma to a group of soft-tissue sarcomas that were often confused with a chronic inflammatory process, a necrotizing granuloma, or a squamous-cell carcinoma ${ }^{2}$. Long term studies showed that $40 \sim 57 \%$ of patients with epithelioid sarcoma developed metastasis ${ }^{3-5}$. The lung is the most common site for dissemination ${ }^{5}$. Other frequent sites include lymph node and scalp $3,5,6$. The trunk, perineum, spine, penis, and vulva also have been occasionally reported as other sites of metastasis ${ }^{6}$.

Address for correspondence: Young Whan Kim, M.D., Ph.D. Division of Pulmonary and Critical Care Medicine, Department of Internal Medicine and Lung Institute of Medical Research Center, Seoul National University College of Medicine, 28, Yeongeon-dong, Jongno-gu, Seoul 110-744, Korea Phone: 82-2-2072-2856, Fax: 82-2-762-9662

E-mail: ywkim@snu.ac.kr

Received: Jul. 8, 2010

Accepted: Sep. 24, 2010
Although the frequency of endobronchial metastasis varies according to definition (from $2 \%$ to $28 \%$ ) $^{7,8}$, the incidence of clinically significant metastasis of major bronchus from extrathoracic tumors occurs in $2 \%$ to $5 \%$ of all cases ${ }^{7}$. The common extrathoracic malignancies include breast, colorectal, and renal carcinoma ${ }^{7}$. There have been only a few reports on endobronchial metastasis from soft tissue sarcoma ${ }^{9,10}$. Especially, there has been no report on endobronchial metastasis from epithelioid sarcoma to our knowledge. This is the first report on epithelioid sarcoma with endobronchial metastasis diagnosed by bronchoscopic and muscle biopsy.

\section{Case Report}

A 28-year-old man presented with chest pain and dyspnea in June 2008. Simple chest radiography and chest computed tomography (CT) revealed bilateral pneumothorax. He underwent bilateral wedge resection by Video-Assisted Thoracoscopic Surgery (VATS) and the histologic diagnosis was 'ruptured alveolar septa 
SY Kim et al: Endobronchial metastasis of epithelioid sarcoma

with granulomatous inflammation'. He underwent right thoracotomy in May 2009 due to recurred pneumothorax. Histologic examination of the lung, pleura and multiple lymph nodes showed only chronic inflammation with granuloma. His physician couldn't identify the cause for recurrent pneumothorax.

In July 2009, he presented with chest pain and dyspnea to Seoul National University Hospital. He complained of hemoptysis, 3 month history of chronic cough, and 2 week history of swelling of left thigh. On physical examination, breath sound was decreased over left lung field and several small, hard, painless nodules in the left thigh were noticed. Routine laboratory test was unremarkable. Chest CT on admission showed left pneumothorax, multiple air cysts and nodules in both lungs and endobronchial lesion in right lower basal lobe (Figure 1A). Serial outside CT images revealed that the endobronchial lesion developed newly in March 2009 and has progressed. The multiple lung nodules have aggravated since 2008. Bronchoscopy showed hemorrhagic mass that obstructed the bronchus of right lower basal lobe (Figure 2). Magnetic resonance imaging revealed multiple nodular lesions of variable size in the left thigh muscles. The specimen of bronchoscopic biopsy and left thigh muscle biopsy were composed of relatively uniform, polygonal or epithelioid cells with abundant, deeply eosinophilic cytoplasm, suggesting epithelioid sarcoma (Figure 3).

Immunohistochemical staining for cytokeratin, epithelial membrane antigen, vimentin, and CD34 were all positive (Figure 4), however, S-100, and CD68 were negative. Finally, he was diagnosed with epithelioid sarcoma.

The patient underwent six cycles of combination che-
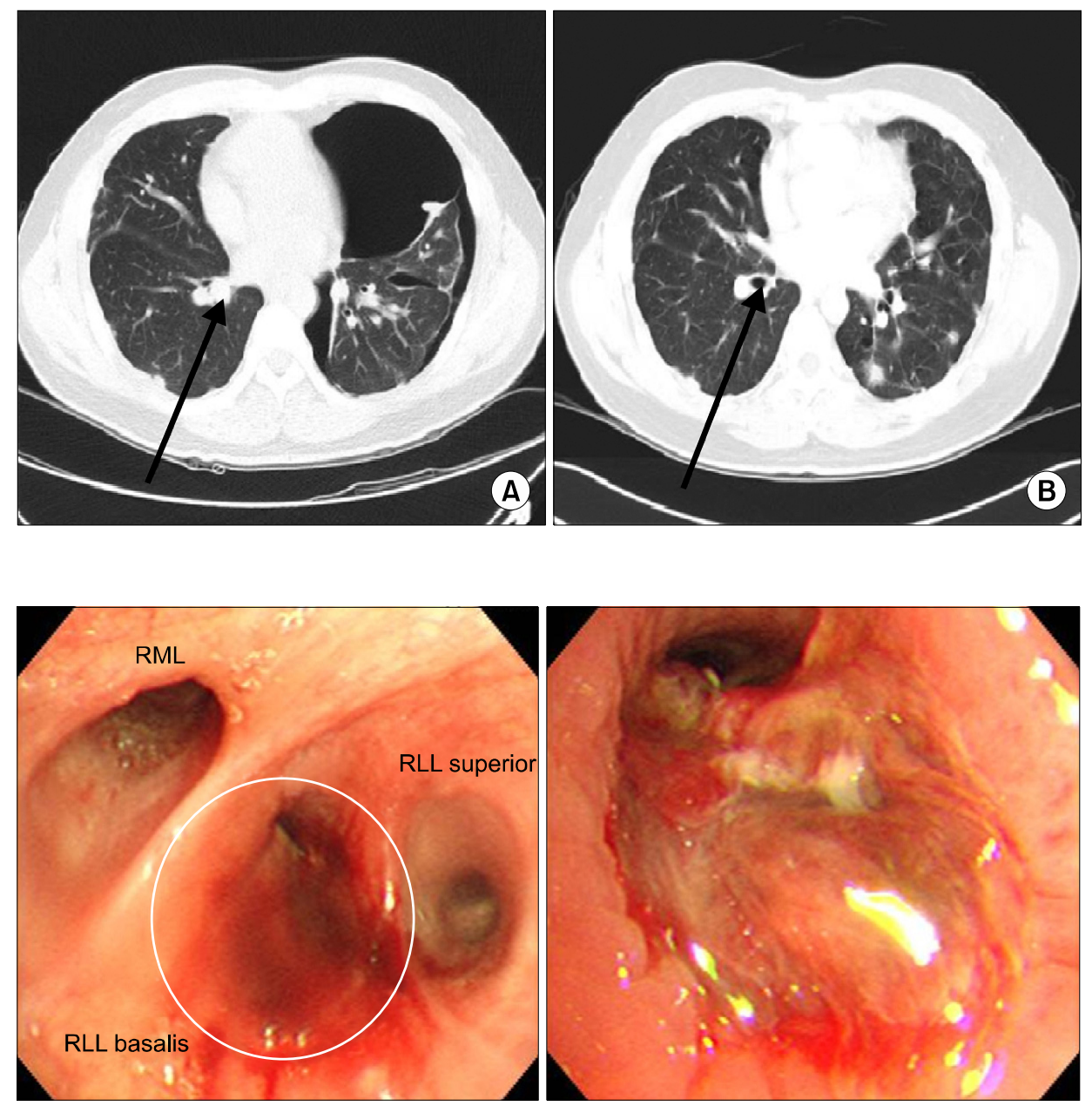

Figure 1. Chest computed tomography. (A) The arrows indicate the endobronchial lesion of right lower lung basalis bronchus. CT shows multiple lung nodules at both lung and left pneumothorax. (B) After chemotherapy, the endobronchial lesion is disappeared.

Figure 2. Fiberobronchoscopic photography showing a endobronchial mass at the orifice of the right lower lobe basalis bronchus. RML: right middle lobe, RLL: right lower lobe. 


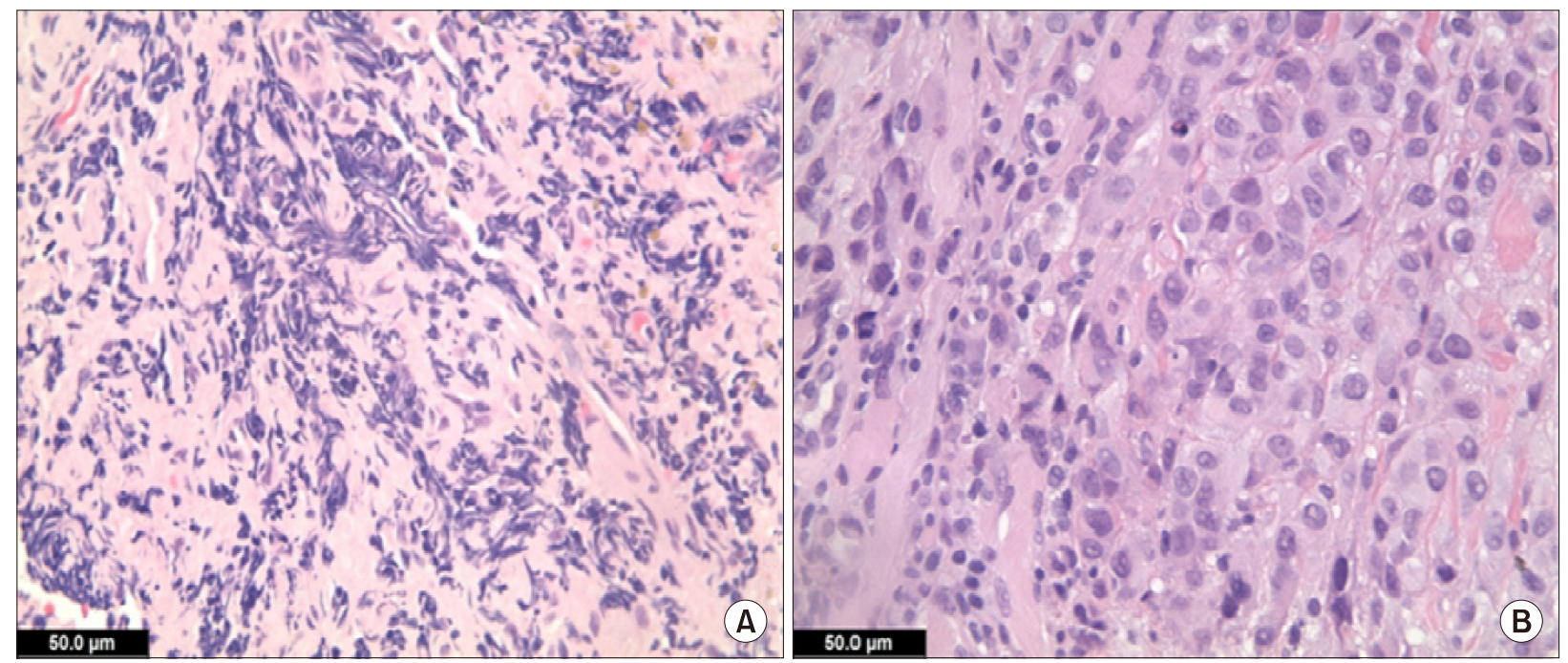

Figure 3. (A) Bronchoscopic biopsy specimen. (B) Muscle biopsy specimen. The specimens were composed of relatively uniform, polygonal or epithelioid cells with abundant, deeply eosinophilic cytoplasm (Hematoxylineosin, original magnification $\times 400$ ).
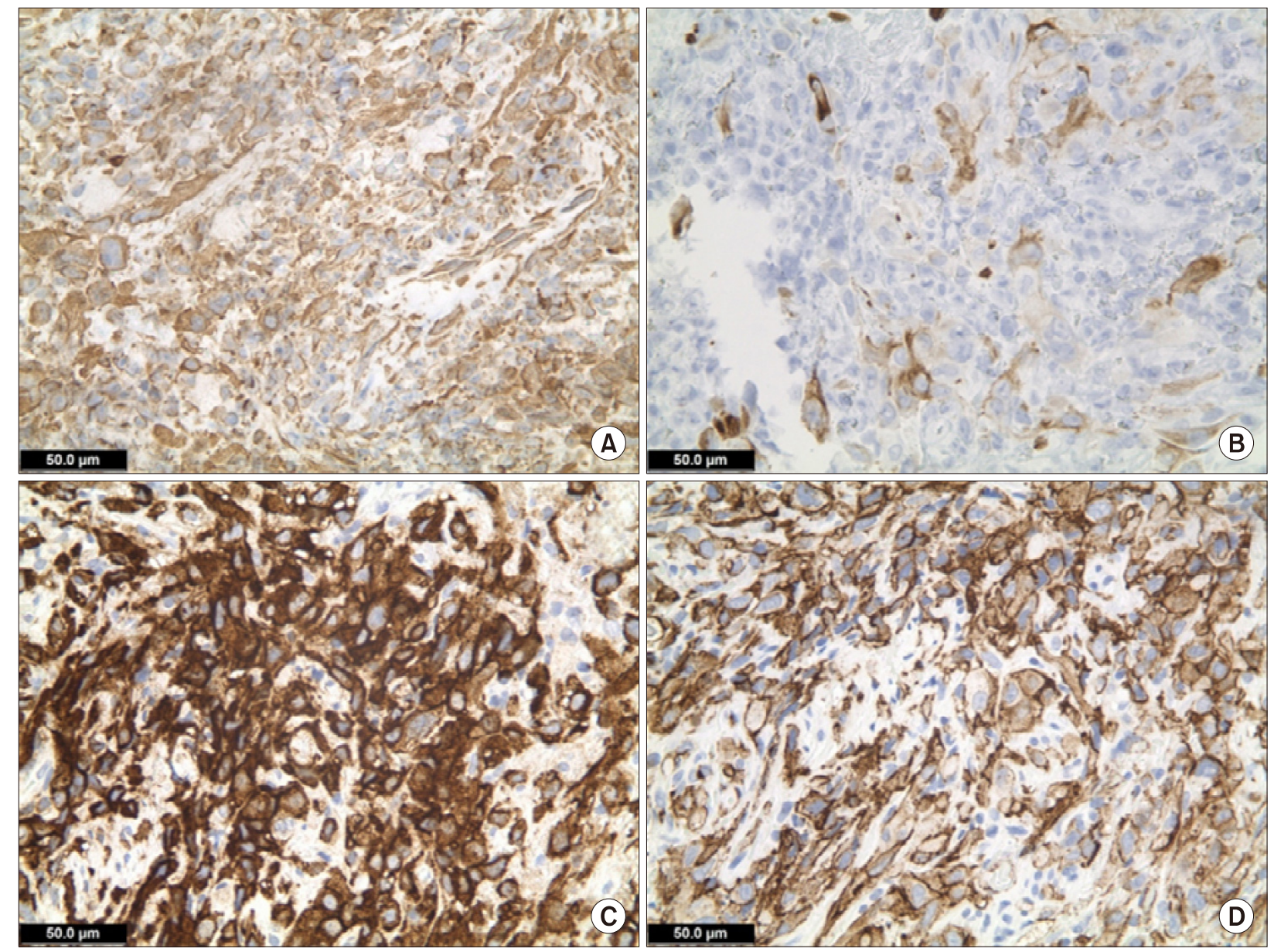

Figure 4. Immunohistochemistry stain by Vimentin (A), Cytokeratin (B), Epithelial Membrane Antigen (C), and CD34 (D) in bronchoscopic biopsy specimen (original magnification $\times 400$ ). 
motherapy with ifosfamide, cisplatin and etoposide from August 2009 to December 2009. While the endobronchial lesion disappeared in chest CT (Figure 1B), the MRI of lower extremity showed progression of the multiple nodules in the left thigh in January 2010. In the left occipital area there was a growing ulcerative lesion, which turned out to be epithelioid sarcoma. Consequently, a progressive disease was diagnosed and the chemotherapy regimen was switched to combination chemotherapy consisting of ifosfamide and adriamycin. He has received additional 4 cycles of chemotherapy so far.

\section{Discussion}

Epithelioid sarcomas are rare subtype of soft tissue sarcoma and have unclear histogenesis. The incidence of epitheloid carcoma is increasing, with a annual percentage change of $5.2 \%$ since 1973 . In 2005, the incidence is estimated 0.4 case per million ${ }^{11}$. Epithelioid sarcoma affects mainly the subcutaneous tissues, fascia, or tendon sheaths of the extremities. There is a preponderance of upper than the lower extremities. Clinically, the tumor usually starts as a painless, slowly growing mass, but not uncommonly presents as a multifocal lesion. The superficial lesions are often ulcerated. Young adults aged between 20 and 40 years old are mostly affected. There is a preponderance for male gender $(2: 1)^{2,12}$.

It is often difficult to diagnose the epithelioid sarcoma. Histologically the sarcoma can be confused with a variety of benign and malignant conditions, including chronic inflammatory processes, granuloma, synovial sarcoma, and ulcerating squamous cell carcinoma ${ }^{2}$. Since the diagnosis is difficult, a definitive diagnosis is often delayed. Ross et al reported that the mean interval between the onset of symptoms and definitive diagnosis was 18 months $^{6}$ and other study showed that the median interval between observing the initial symptoms, making a diagnosis, and starting treatment was 3.5 months, ranging between 1 month and 36 months ${ }^{5}$. In the present case, we needed 13 months from onset of symptom to histological diagnosis of epithelioid sarcoma.

Epitheloid sarcoma is a high grade tumor with a high local recurrence and metastasis rate. The local recurrence rate ranges between 50 70\%,6, and the metastasis rate between $40 \sim 57 \% \%^{3-5}$. Unlike other soft tissue sarcomas $(2.6 \%)$, the rate of lymph node involvement is remarkably high. The common metastasis sites include the lung (51\%) regional lymph nodes (34\%), scalp (22\%), and bone (13\%).

There have been only a few reports on endobronchial metastasis from soft tissue sarcoma including angiosarcomna and leiomyosarcoma ${ }^{9,10}$. Although the lung is the most common site of metastasis, only lung parenchyma is typically involved. An endobronchial metastasis of epithelioid sarcoma has not been reported yet.

Wide surgical resection is recommended as treatment of choice ${ }^{5}$ due to the high local recurrence and metastasis rate of epithelioid sarcoma. High-dose chemoradiotherapy can also achieve low local recurrence rates $^{6,13,14}$. In general, the treatment of endobronhial metastasis is determined by the histology and location of the primary tumor and other metastasis sites, as well as the patients' performance status ${ }^{15}$. The majority of patients with endobronchial metastasis have metastasis to other sites other than endobronchial tree. Therefore most patients are treated with systemic chemotherapy. But in patients with localized disease, surgical resection can be considered ${ }^{15}$.

Our patient has been treated with systemic chemotherapy since he had multiple nodules in the thigh with multiple lung metastasis at the time of diagnosis. Although endobronchial lesion disappeared after six cycles of chemotherapy, lung metastasis increased so that his overall disease status was "progressive disease."

In epithelioid sarcoma, advanced age, male gender, proximal or axial location, depth, tumor size, mitotic figures, necrosis, vascular invasion, and tumor hemorrhage have been identified as adverse prognostic factors $^{11}$. In addition, local recurrence, lymph node metastasis, and the extent of surgery were also associated with survival in some studies ${ }^{6,11,12}$. The 5-year overall 
survival ranges between $60 \%$ and 75\%,3,11 . In summary, we report the first case of epithelioid sarcoma with endobronchial metastasis, confirmed by bronchoscopic biopsy. Although our patient underwent wedge resection of the lung three times, we could not timely diagnose the epitheolid sarcoma with endobronchial metastasis. Because epitheloid sarcoma can be easily confused with chronic inflammatory processes and granuloma, a correct pathologic diagnosis by an experienced pathologist is essential.

\section{References}

1. Cormier JN, Pollock RE. Soft tissue sarcomas. CA Cancer J Clin 2004;54:94-109.

2. Enzinger FM. Epitheloid sarcoma. A sarcoma simulating a granuloma or a carcinoma. Cancer 1970;26: 1029-41.

3. Spillane AJ, Thomas JM, Fisher C. Epithelioid sarcoma: the clinicopathological complexities of this rare soft tissue sarcoma. Ann Surg Oncol 2000;7:218-25.

4. Whitworth PW, Pollock RE, Mansfield PF, Couture J, Romsdahl MM. Extremity epithelioid sarcoma. Amputation vs local resection. Arch Surg 1991;126:1485-9.

5. de Visscher SA, van Ginkel RJ, Wobbes T, Veth RP, Ten Heuvel SE, Suurmeijer AJ, et al. Epithelioid sarcoma: still an only surgically curable disease. Cancer
2006;107:606-12.

6. Ross HM, Lewis JJ, Woodruff JM, Brennan MF. Epithelioid sarcoma: clinical behavior and prognostic factors of survival. Ann Surg Oncol 1997;4:491-5.

7. Braman SS, Whitcomb ME. Endobronchial metastasis. Arch Intern Med 1975;135:543-7.

8. Rosenblatt MB, Lisa JR, Trinidad S. Pitfalls in the clinical histologic diagnosis of bronchogenic carcinoma. Dis Chest 1966;49:396-404.

9. Udelsman R, Roth JA, Lees D, Jelenich SE, Pass HI. Endobronchial metastases from soft tissue sarcoma. J Surg Oncol 1986;32:145-9.

10. Warren WH, Bleck P, Kittle CF, Faber LP. Surgical management of pulmonary metastatic leiomyosarcoma with gross endobronchial extension. Ann Thorac Surg 1990; 50:739-42

11. Jawad MU, Extein J, Min ES, Scully SP. Prognostic factors for survival in patients with epithelioid sarcoma: 441 cases from the SEER database. Clin Orthop Relat Res 2009;467:2939-48.

12. Bos GD, Pritchard DJ, Reiman HM, Dobyns JH, Ilstrup DM, Landon GC. Epithelioid sarcoma. An analysis of fifty-one cases. J Bone Joint Surg Am 1988;70:862-70.

13. Shimm DS, Suit HD. Radiation therapy of epithelioid sarcoma. Cancer 1983;52:1022-5.

14. Armah HB, Parwani AV. Epithelioid sarcoma. Arch Pathol Lab Med 2009;133:814-9.

15. Salud A, Porcel JM, Rovirosa A, Bellmunt J. Endobronchial metastatic disease: analysis of 32 cases. J Surg Oncol 1996;62:249-52. 